\title{
Anisotropic surface coupling while sliding on dolomite and calcite crystals
}

\author{
Carlos M. Pina, ${ }^{1}$ Rodolfo Miranda, ${ }^{2,3}$ and Enrico Gnecco ${ }^{2}$ \\ ${ }^{1}$ Department of Crystallography and Mineralogy, Complutense University of Madrid, E-28040 Madrid, Spain \\ ${ }^{2}$ Instituto Madrileño de Estudios Avanzados, IMDEA Nanociencia, Campus Universitario de Cantoblanco, E-28049 Madrid, Spain \\ ${ }^{3}$ Departamento de Física de la Materia Condensada, Universidad Autónoma de Madrid, Cantoblanco, 28049 Madrid, Spain
}

(Received 21 December 2011; published 6 February 2012)

\begin{abstract}
High-resolution friction force microscopy has been performed on the (104) surfaces of dolomite and calcite in deionized water. The two rows of oxygen atoms alternating in a zigzag way on top of both surfaces are resolved with similar contrast while scanning along the $[42 \overline{1}]$ direction. Along the [010] direction, only one row is resolved, provided that the normal loading is large enough. The direction-dependent interaction between the probing tip and surface atoms is explained by numeric calculations based on the Prandtl-Tomlinson model.
\end{abstract}

DOI: 10.1103/PhysRevB.85.073402

PACS number(s): 68.35.Af, 07.79.Sp, 91.60.Ed

With the exception of a few noticeable superlubric effects, ${ }^{1}$ the sliding of two solid surfaces past each other is accompanied by stick-slip events due to the continuous formation and rupture of interatomic bonds. ${ }^{2}$ On geological scales stick-slip is responsible for earthquakes and notoriously difficult to predict. The scenario radically changes at the nanoscale, where the loading and friction forces on sharp tips terminated by just a few atoms can be easily controlled and monitored by atomic force microscopy (AFM) in contact mode. ${ }^{3}$ In the first approximation, the probing tip of an AFM can be considered a point mass driven by a lateral spring. This spring is associated with the torsion of a cantilever beam supporting the tip combined with the shear deformation of the contact region. ${ }^{4}$ On a crystal surface the tip sticks to a lattice site till the spring elongation reaches a critical value and the tip suddenly jumps and binds again to another site in a stick-slip fashion. The friction forces developed in this process peak while scanning along a main crystallographic direction, the peak intensity being determined by the density of packing of the surface atoms. ${ }^{5}$ Systematic measurements of such "friction anisotropy" were reported on highly oriented pyrolitic graphite, ${ }^{6}$ metallic quasicrystals, ${ }^{7}$ and organic crystals. ${ }^{8}$ However, the results of the first study could only be explained by assuming the presence of a graphite flake attached to the tip apex, the second investigation did not report any friction maps on the atomic scale, and the third one did not reveal sublattice features in the complex surface structure. This was indeed possible on another organic crystal, where Fessler et al. were recently able to distinguish between two differently oriented molecules forming the unit cell of a surface lattice, although the direction of motion of the tip was kept fixed. ${ }^{9}$

In this article we have chosen the (104) cleavage surfaces of dolomite and calcite as prototype systems for studying the influence of the sliding direction on atomic stick-slip on surfaces of intermediate crystallochemical complexity. Mainly due to their interest for earth and environmental sciences, calcite and dolomite (104) surfaces have been extensively studied with numerous surface sensitive techniques. ${ }^{10-12}$ However, their tribological properties have scarcely been investigated. ${ }^{13}$ The structure of the two surfaces is shown in Fig. 1. In both cases a

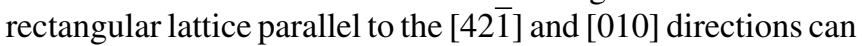
be defined. The unit cell of the lattice has dimensions of $a=$ $0.771 \mathrm{~nm}$ and $b=0.481 \mathrm{~nm}$ for dolomite and $a=0.810 \mathrm{~nm}$ and $b=0.499 \mathrm{~nm}$ for calcite. In each cell two oxygen atoms protrude out of the surface. These atoms alternate in a zigzag way along the $[42 \overline{1}]$ direction, whereas they are aligned along the [010] direction. Hereafter, these atoms are referred to as oxygens of types I and II, according to their position in Fig. 1. Furthermore, two calcium atoms per unit cell appear aligned along the [421] direction on calcite (104). On dolomite (104) one of the $\mathrm{Ca}$ atoms is ideally replaced by $\mathrm{Mg}$. Here we show how, depending on the scan direction, either one or two lattice sites per unit cell are revealed in the friction maps acquired by AFM on these surfaces. These sites are unambiguously related to the protruding oxygens. This means that the coupling between tip and surface can be modified by varying the sliding direction. This intriguing phenomenon can be explained with the classical Prandtl-Tomlinson (PT) model, which has been repeatedly used to reproduce friction maps on different systems including $\mathrm{NbSe}_{2},{ }^{14}$ graphite, ${ }^{15-17}$ $\mathrm{KBr},{ }^{18} \mathrm{NaF},{ }^{19} \mathrm{MoS}_{2},{ }^{20}$ and $\beta-\mathrm{MoTe}_{2}(001) .{ }^{21}$

Our measurements were performed at room temperature using a commercial AFM (Multimode Veeco Instruments) equipped with a fluid cell. To minimize adhesion forces during measurements, surfaces were immersed in deionized water (Milli-Q Millipore; resistivity, $18 \mathrm{M} \Omega \mathrm{cm}$ ). Crystals used were dolomite from Eugui (Spain) and calcite from Durango (Mexico), both of optical quality. Crystals were cleaved with a blade razor along their (104) faces immediately prior to being placed in the fluid cell of the AFM. Silicon nitride triangular tips (Veeco DNP-S10 and SNL-10) were used. Friction forces were calibrated using the method introduced by Noy et al..$^{22}$ and the analysis of all the AFM images collected was performed with the WSxM 4.0 software. ${ }^{23}$ High-resolution topography images of dolomite (104) were recently reported using the same setup. ${ }^{24}$

Figure 2 shows a topography image and the corresponding friction map of a dolomite (104) surface on the microscale, immediately after cleaving and placing it in the fluid cell of the AFM. A significant increase in friction is found when crossing the step edges upward, which is similar to what is observed on cleavage steps of various crystal surfaces under ambient conditions ${ }^{25}$ and in ultrahigh vacuum. ${ }^{26}$ As the next step, we acquired hundreds of friction images on the flat terraces of dolomite (104) with a scan range of a few nanometers. The sample was rotated by different angles, with the scan direction always perpendicular to the cantilever beam. The normal force 

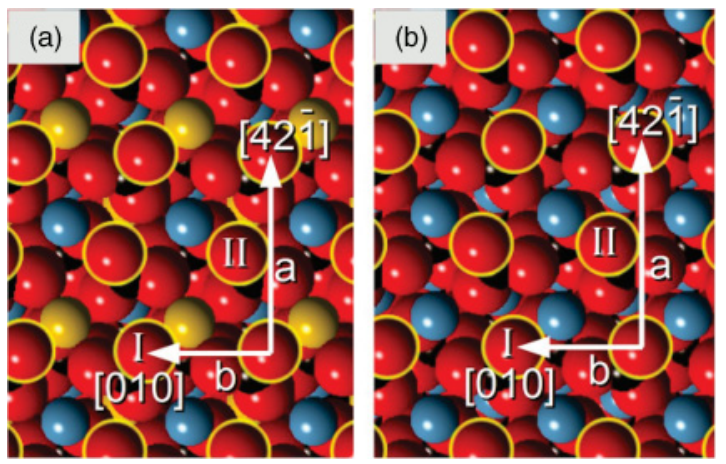

FIG. 1. (Color online) Structure of the (104) cleavage surfaces of (a) dolomite and (b) calcite. Oxygen atoms are represented by red spheres, carbon atoms by black spheres, calcium atoms are blue, and magnesium atoms are yellow. Oxygen atoms enhanced by yellow outlines are protruding from the surface. Depending on their arrangement, these oxygens can be distinguished as two types.

$F_{N}$ was varied up to $65 \mathrm{nN}$. In Fig. 3(a) the scan direction

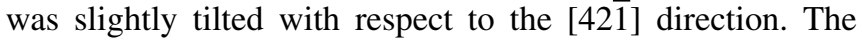
rectangular unit cell of the surface lattice is easily recognized. In each cell two friction peaks of different intensities can be observed. This is better seen after averaging over the cells (see top right corner). From a comparison with the crystal structure in Fig. 1(a), we can univocally attribute the peaks in the friction map to the rupture of "bonds" formed between the tip apex and the protruding oxygens of the dolomite surface while scanning. A strong interaction of the tip apex with $\mathrm{Mg}$ and/or e Ca atoms would give rise to simple rectangular structures, which are not compatible with Fig. 3(a). Furthermore, the intensity and size of the frictional peaks are not the same. Two types of contrast can be distinguished, which reflect the different arrangements of the protruding oxygens in Fig. 1(a). The main peaks correspond to oxygen atoms of type I when the tip is scanned from left to right and to type II when scanned from right to left (pink inset). This contrast is much more pronounced when scanning close to the $[0 \overline{1} 0]$ direction [Fig. 3(b)]. In this case the minor peaks tend to vanish and only one frictional peak per unit cell is essentially observed.

In order to understand the strong anisotropy effects shown in Fig. 3, we simulated the imaging process using the PT model. Calculations were performed in adimensional units with the same procedure used in a recent work on hexagonal lattices. ${ }^{5}$ The tip-surface interaction was reproduced with a periodic
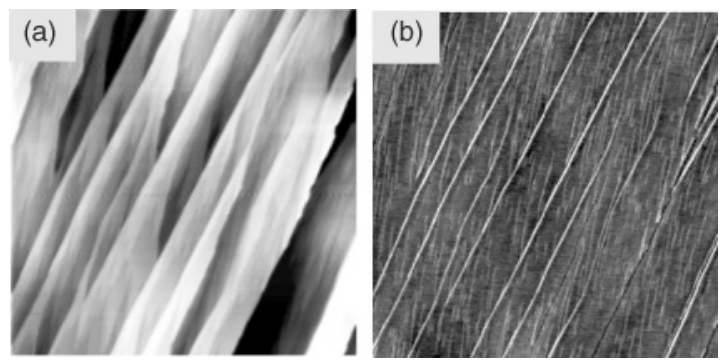

FIG. 2. (a) AFM topography and (b) friction map of a dolomite (104) surface in water. Frame size: $33 \times 3 \mu \mathrm{m}^{2}$. Scan direction: left to right.

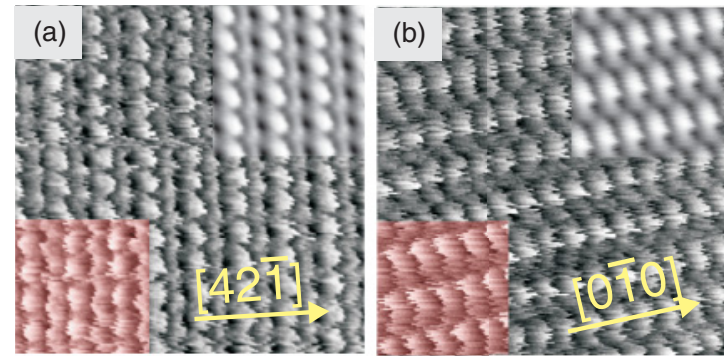

FIG. 3. (Color online) Friction maps of dolomite (104) acquired with a misalignment of (a) $-4^{\circ}$ from the $[42 \overline{1}]$ direction and (b) $-12^{\circ}$ from the [010] direction. The normal force is $F_{N}=11 \mathrm{nN}$ in both cases, corresponding to lateral (friction) forces $F_{L}=12$ and $18 \mathrm{nN}$, respectively. The top-right portions of these maps have been averaged over the surface lattice. Insets (pink) at the lower left correspond to friction along the opposite directions and show the inversion of the row-pairing effect discussed in the text. Image size: $6.5 \times 6.5 \mathrm{~nm}^{2}$.

potential $V(x, y)$ having symmetry of the protruding oxygens in the dolomite (104) surface [Fig. 4(a)]. A potential well with a close-to-Gaussian shape was associated with each of these atoms. The profile of the potential well was assumed to be the same for both type I and type II oxygens, i.e., the influence of the surrounding atoms on the tip-surface interaction was supposed to be negligible. In formulas, $V(x, y)=\sum_{i=1}^{2} V_{i}$, where

$$
V_{i}(x, y)=-\eta \exp \left[-\left(\frac{\sin ^{2} \beta\left(x-x_{i}\right)}{\beta^{2} r^{2}}+\frac{\sin ^{2}\left(y-y_{i}\right)}{r^{2}}\right)\right] .
$$

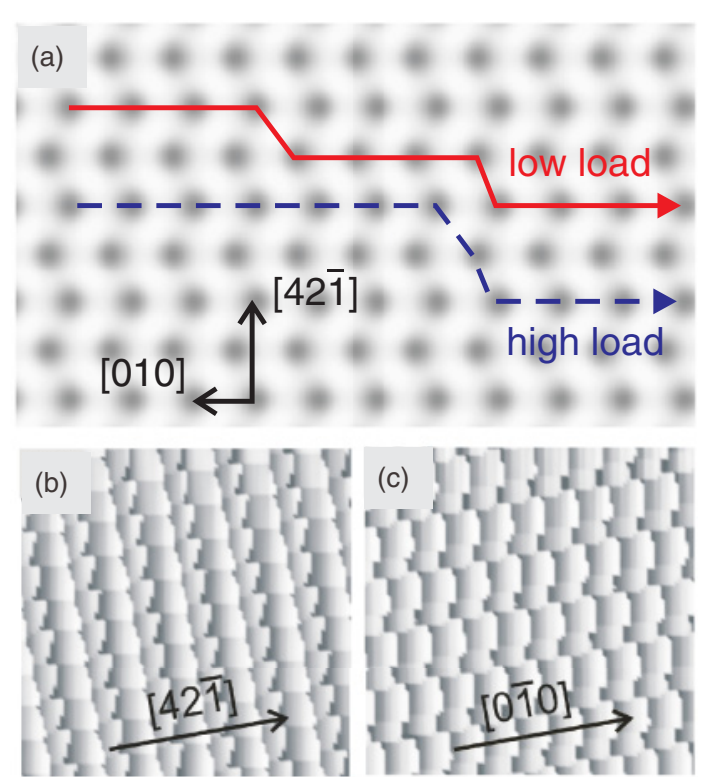

FIG. 4. (Color online) (a) Tip-surface interaction potential, (1), with parameter values $\eta=3$ and $r=1$. Minima correspond to protruding oxygen atoms of the dolomite (104) surface. The solid (red) curve shows a tip trajectory obtained while scanning at $-10^{\circ}$ from the $[0 \overline{1} 0]$ direction. The dashed (blue) curve replaces it when $\eta=10$. (b, c) Corresponding friction maps when scanning at $-10^{\circ}$ from the $[42 \overline{1}]$ and $[0 \overline{1} 0]$ directions, respectively. 
In Eq. (1) the coordinates of the protruding atoms in the unit cell are given by $\left(x_{1}, y_{1}\right)=(0,0)$ and $\left(x_{2}, y_{2}\right)=(3 \pi / 4, \pi / 2)$, whereas $\beta=1.6$ is the ratio between the lattice constants along

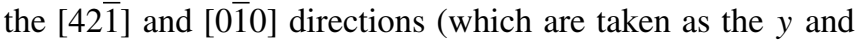
$x$ axes, respectively). With this choice, the potential wells alternate in two rows shifted by $a / 2$ along the $y$ direction and by $3 b / 4$ along the $x$ direction. This is to reproduce the fact that the relative position of the protruding oxygens is $0.496 \times a$ and $0.745 \times b$ for dolomite and $0.500 \times a$ and $0.742 \times b$ for calcite. The parameter $r$ defines the width of the potential wells, whereas $\eta$ quantifies the strength of the tip-surface interaction compared to the elastic energy stored in the lateral spring and generally increases with the normal force $F_{N} \cdot{ }^{27}$ If the load is not too high $(\eta=3)$, both types of protruding oxygens are clearly distinguished (with different contrast) when scanning close to the [42 1 ] direction [Fig. 4(b)]. The contrast is significantly increased when scanning close to the [010] direction [Fig. 4(c)] and only atoms of type I are clearly resolved in the friction map.

To gain more insight we also estimated the pathway of the tip apex on the surface lattice. At low loads [solid (red) curve in Fig. 4(a)], the tip binds to both types of oxygens, although it preferentially sticks at type I locations (as seen by examining a long series of jumps with different offsets in the slow scan direction). Upon an increase in the load $(\eta=10)$, oxygens of type II are avoided by the tip, which couples only to oxygens of type I [dashed (blue) curve]. This is related to the fact that, due to the asymmetric arrangement of the minima in the potential, (1), larger energy barriers must be overcome to reach locations of type II once the parabolic potential describing the lateral deformation of the sliding contact in the PT model is taken into account. It is interesting to observe that the influence of the load on the number of friction peaks per unit cell has already been reported by other groups. On the $\beta-\mathrm{MoTe}_{2}(001)$ surface, Te atoms align in two rows of different heights, and, as recognized by Hölscher et al., the lower row is hindered if the normal force is not large enough. ${ }^{21}$ On $\mathrm{NaF}(001)$ one of the two ionic species could be revealed only in a limited range of low loading forces. ${ }^{28,29}$ Conversely, large loads may cause jumping across several lattice constants and the apparent "disappearance" of unit cells. ${ }^{30}$ In this article, this kind of effect is coupled to the direction of sliding with respect to the anisotropic arrangement of the surface atoms.

Further support for our analysis came from friction measurements on calcite (104). The frictional response of this surface is indeed very similar to that of dolomite (104). In

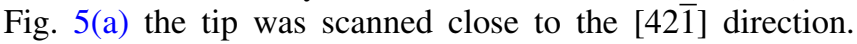
Both types of oxygen atoms are resolved and the two species look similar. When scanning close to the [010] direction, a strong contrast between the I and the II species again appears [Fig. 5(b)]. The previous interpretation based on the PT model can thus be extended to the calcite (104) surface, confirming that $\mathrm{Ca}$ atoms (and also $\mathrm{Mg}$ atoms in the case of dolomite) do not play any important role in the sliding process. We note that, in the case of calcite, coupling of the AFM tip to the protruding oxygens has been suggested by various authors without measuring friction forces. ${ }^{31-34}$ Topography images showing alternate heights of oxygen atoms were presented by Stipp et al. "1 who introduced the term "row pairing" to describe this effect. Although it was postulated that row

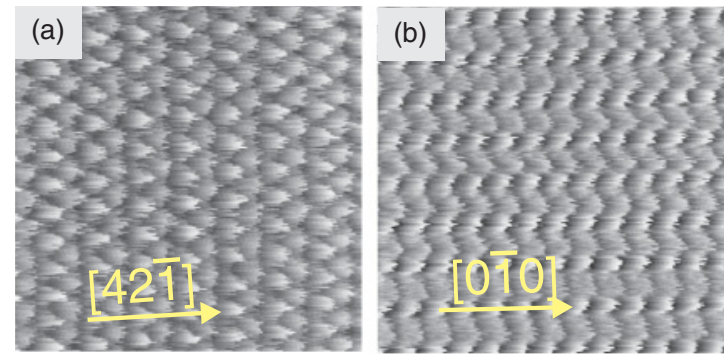

FIG. 5. (Color online) Friction maps of calcite (104) with a misalignment of (a) $-4^{\circ}$ from the $[42 \overline{1}]$ direction and (b) $-2^{\circ}$ from the [010] direction. The normal force was $F_{N}=29$ and $12 \mathrm{nN}$, respectively, corresponding to lateral forces $F_{L}=35$ and $17 \mathrm{nN}$. Image size: $6.5 \times 6.5 \mathrm{~nm}^{2}$.

pairing results from the asymmetry of the scanning process, ${ }^{35}$ experimental evidence and modeling of this phenomenon have been missing up to now. Our measurements strongly suggest that the different pulling forces acting on the tip bound to oxygens of types I and II are responsible for the row pairing. We should mention that in a few cases we did not observe any inversion in the row-pairing effect. When sliding back and forth along the [010] direction of calcite, one series of measurements showed friction peaks corresponding to only one type of oxygen atoms. This may be due to the temporary transfer of material to the tip apex, which introduced a structural asymmetry independent of the scan direction.

As the last step we measured the load dependence of friction on both calcite and dolomite (104). Force-distance curves showed almost no adhesion on either surface, as is typical in liquid environments. ${ }^{31}$ On dolomite (104), friction was found to increase up to $45 \pm 10 \mathrm{nN}$ at the maximum normal force applied $\left(F_{N}=65 \mathrm{nN}\right)$, which is consistent with previous measurements by Higgins et al. ${ }^{10}$ On calcite (104), higher friction (up to $65 \pm 15 \mathrm{nN}$ when $F_{N}=65 \mathrm{nN}$ ) was found. The scatter of the experimental data, reflecting possible variations in the structure of the tip apex, did not allow us to distinguish between a linear dependence of friction on the normal force and the $2 / 3$ power law expected from continuous mechanics. ${ }^{36}$ In any case, on both calcite and dolomite (104), significant variations in the mean values of the friction force along the [010] versus the [42 $\overline{1}]$ direction could not be observed. Once again, this is in agreement with the PT model. By averaging simulated friction maps like those in Figs. 4(b) and 4(c), we did not observe significant differences between the two sliding directions, despite the strong contrast shown in the images.

Note that the exact nature of the tip-surface interaction is not discussed in this article. On one hand, the probing tip can easily pick up surface atoms while scanning in contact mode. This process can be enhanced by dissolution of the dolomite and calcite surfaces, which release calcium atoms in water. ${ }^{11,37}$ Strong chemical bonds could thus be formed between $\mathrm{Ca}$ atoms adhering to the tip apex and $\mathrm{O}$ atoms protruding out of the surface. On the other hand, relaxation of the silicon nitride tip apex in the hollow sites of the interaction potential without strong perturbations of the electronic states is also compatible with the stick-slip behavior observed in our experiments. Due to the lack of adequate atomistic simulations, it is difficult to conclude which of the two scenarios is more likely. 
In conclusion, we have observed that the interaction between an atomically sharp tip and dolomite and calcite surfaces is strongly affected by the direction of the tip motion. The row pairing of oxygen atoms, previously reported in the literature, has been related to the tip pathway as predicted by the PT model. Row-pairing inversion is expected and observed when the sliding direction is reversed. Due to their intermediate complexity, calcite and dolomite act as model systems for investigating this anisotropy effect, which is, in principle, not limited to those mineral surfaces. The fact that the coupling between a sharp tip and a crystal surface can be promoted or hindered by varying the sliding direction must be taken into account when dealing with friction on the nanoscale and could be successfully used to control the manipulation of tiny objects on the nanometer scale.

We would like to thank Dr. Reinhold Wannemacher and Professor Alexis Baratoff for stimulating discussions on this topic. Atomic force microscopy measurements were performed at the ICTS Centro Nacional de Microscopía Electrónica, Madrid. *enrico.gnecco@imdea.org

${ }^{1}$ A. Erdemir and J. M. Martin (eds.), Superlubricity (Elsevier, Amsterdam, 2007).

${ }^{2}$ F. P. Bowden and D. Tabor, The Friction and Lubrication of Solids (Clarendon Press, Oxford, 1954).

${ }^{3}$ B. Bhushan (ed.), Springer Handbook of Nanotechnology, 3rd ed. (Springer-Verlag, Berlin, 2010).

${ }^{4}$ R. W. Carpick, D. F. Ogletree, and M. Salmeron, Appl. Phys. Lett. 70, 1548 (2010).

${ }^{5}$ E. Gnecco, Europhys. Lett. 91, 66008 (2010).

${ }^{6}$ M. Dienwiebel, G. S. Verhoeven, N. Pradeep, J. W. M. Frenken, J. A. Heimberg, and H. W. Zandbergen, Phys. Rev. Lett. 92, 126101 (2004).

${ }^{7}$ J. Y. Park, D. F. Ogletree, M. Salmeron, R. A. Ribeiro, P. C. Canfield, C. J. Jenks, and P. A. Thiel, Science 309, 1354 (2005).

${ }^{8}$ M. Campione and E. Fumagalli, Phys. Rev. Lett. 105, 166103 (2010).

${ }^{9}$ G. Fessler, I. Zimmermann, T. Glatzel, E. Gnecco, P. Steiner, R. Roth, T. D. Keene, S. X. Liu, S. Decurtins, and E. Meyer, Appl. Phys. Lett. 98, 083119 (2011).

${ }^{10}$ S. R. Higgins, X. Hu, and P. Fenter, Langmuir 23, 8909 (2007).

${ }^{11}$ S. L. S. Stipp, C. M. Eggleston, and B. S. Nielsen, Geochim. Cosmochim. Acta 58, 3023 (1994).

${ }^{12}$ J. Schütte, P. Rahe, L. Tröger, S. Rode, R. Bechstein, M. Reichling, and A. Kühnle, Langmuir 26, 8295 (2010).

${ }^{13}$ P. Cubillas and S. R. Higgins, Geochem. Trans. 10, 7 (2009).

${ }^{14}$ J. W. J. Kerssemakers and J. T. M. De Hosson, Appl. Phys. Lett. 67, 347 (1995).

${ }^{15}$ N. Sasaki, K. Kobayashi, and M. Tsukada, Phys. Rev. B 54, 2138 (1996).

${ }^{16}$ U. von Toussiant, Th. Schimmel, and J. Küppers, Surf. Interface Anal. 25, 620 (1997).

${ }^{17}$ H. Hölscher, U. D. Schwarz, O. Zwörner, and R. Wiesendanger, Phys. Rev. B 57, 2477 (1998).

${ }^{18}$ R. Lüthi, E. Meyer, M. Bammerlin, L. Howald, H. Haefke, T. Lehmann, C. Loppacher, and H. J. Güntherodt, J. Vac. Sci. Technol. B 14, 1280 (1996).
${ }^{19}$ H. Hölscher, U. D. Schwarz, and R. Wiesendanger, Europhys. Lett. 36, 19 (1996).

${ }^{20}$ H. Hölscher, U. D. Schwarz, and R. Wiesendanger, Surf. Sci. 375, 395 (1997).

${ }^{21}$ H. Hölscher, W. Raberg, U. D. Schwarz, A. Hasbach, K. Wandelt, and R. Wiesendanger, Phys. Rev. B 59, 1661 (1999).

${ }^{22}$ A. Noy, C. D. Frisbie, L. F. Rozsnyai, M. S. Wrighton, and C. M. Lieber, J. Am. Chem. Soc. 117, 7943 (1995).

${ }^{23}$ I. Horcas, R. Fernandez, J. M. Gomez-Rodriguez, J. Colchero, J. Gomez-Herrero, and A. M. Baro, Rev. Sci. Instrum. 78, 013705 (2007).

${ }^{24}$ C. M. Pina, C. Pimentel, and M. García-Merino, Surf. Sci. 604, 1877 (2010).

${ }^{25}$ H. Hölscher, D. Ebeling, and U. D. Schwarz, Phys. Rev. Lett. 101, 246105 (2008)

${ }^{26}$ P. Steiner, E. Gnecco, F. Krok, J. Budzioch, L. Walczak, J. Konior, M. Szymonski, and E. Meyer, Phys. Rev. Lett. 106, 186104 (2011)

${ }^{27}$ A. Socoliuc, R. Bennewitz, E. Gnecco, and E. Meyer, Phys. Rev. Lett. 92, 134301 (2004).

${ }^{28}$ S. Fujisawa, Y. Sugawara, and S. Morita, Philos. Mag. A 74, 1329 (1996).

${ }^{29}$ M. Ishikawa, S. Okita, N. Minami, and K. Miura, Surf. Sci. 445, 488 (2000).

${ }^{30}$ S. N. Medyanik, W. K. Liu, I. H. Sung, and R. W. Carpick, Phys. Rev. Lett. 97, 136106 (2006).

${ }^{31}$ F. Ohnesorge and G. Binnig, Science 260, 1491 (1993).

${ }^{32}$ Y. Liang, A. S. Lea, D. R. Baer, and M. H. Engelhard, Surf. Sci. 351, 172 (1996).

${ }^{33}$ S. Rode, N. Oyabu, K. Kobayashi, H. Yamada, and A. Kühnle, Langmuir 23, 2850 (2009).

${ }^{34}$ S. Rode, N. Oyabu, K. Kobayashi, H. Yamada, and A. Kühnle, Langmuir 26, 8295 (2010).

${ }^{35}$ S. L. S. Stipp, Geochim. Cosmochim. Acta 63, 3121 (1999).

${ }^{36} \mathrm{X}$. Hu, P. Cubillas, and S. R. Higgins, Langmuir 26, 4769 (2010).

${ }^{37}$ X. Hu, D. A. Grossie, and S. R. Higgins, Am. Mineral. 90, 963 (2005). 